\title{
On the tangibility of letters and braille
}

\author{
JACK M. LOOMIS \\ University of California, Santa Barbara, California 93106
}

\begin{abstract}
Braille characters and letters were presented to the senses of touch and vision of sighted observers. The optical stimuli were subjected to low-pass spatial filtering to the degree that visual spatial resolution closely matched tactile spatial resolution (re the size of characters presented). In one experiment, three sets of braille characters and five sets of Roman letters, varying in typography, were presented as stimuli. The variations in tangibility and legibility across the eight character sets were highly correlated $(r=.98)$. In a second experiment, braille characters and letters were varied in size. The psychometric functions relating tangibility and legibility to character size were similar in both shape and position along the abscissa. The congruence of tangibility and legibility for these conditions, in which the senses were matched in spatial resolution, supports the idea that braille characters are more tangible by virtue of their greater distinctiveness after the low-pass spatial filtering of cutaneous processing.
\end{abstract}

Over the last century and a half, braille has evolved into an effective medium for touch reading (Bledsoe 1972; Bürklen, 1932; Lowenfeld, Abel, \& Hatlen, 1969; Merry, 1937; Nolan \& Kederis, 1969), but understanding of its effectiveness has not been forthcoming. Most research on braille has been largely atheoretical, concerned with such things as the relative tangibility of the different characters (Bürklen, 1932; Nolan \& Kederis, 1969), the effects of character and line spacing on reading rate (Meyers, Ethington, \& Ashcroft, 1958), and the factors influencing word recognition thresholds (Nolan \& Kederis, 1969). An overview of the research on braille is provided in the monograph by Nolan and Kederis (1969).

The present work addresses the question of why braille characters are more tangible than embossed Roman letters, an accepted fact about braille (Bledsoe, 1972; Merry, 1937). The hypothesis being considered here is that braille characters are more varied in terms of their lower spatial frequency content than upperor lowercase letters of the same size and that, by virtue of the limited spatial resolution of the cutaneous sense, they give rise to more differentiated patterns of neural activity in the somatosensory projection areas than do letters. ${ }^{1}$ It is stated as part of the hypothesis, rather than as fact, that braille characters vary more than letters in terms of low spatial frequency content. It might seem an easy matter to show the truthfulness of the statement by simply determining the physical similarity of the letters and braille characters after spatial frequency filtering. However, the concept of physical similarity is not well defined; there are a

This work was presented at the 1979 meeting of the Psychonomic Society in Phoenix, Arizona. It was supported by Grant 1 RO1 NS 15129 from NINCDS and by the Academic Senate, University of California, Santa Barbara. The author thanks Kenneth Pulliam, John Foley, and a reviewer for useful comments on earlier versions of the manuscript. multitude of ways in which one might choose to define it. True, it may be that, for a wide range of definitions, braille characters could be shown to be more dissimilar (and hence discriminable) than letters. Even so, it would be necessary to conduct experiments on tactile recognition to link the measures of similarity to the facts of recognition. The strategy adopted in this work was, instead, to determine whether the tactile recognition of letters and braille characters could be mimicked by visual recognition of the same characters after having been subjected to an appropriate degree of low-pass spatial filtering. This strategy has been used previously with some success (Apkarian-Stielau \& Loomis, 1975; Loomis \& Apkarian-Stielau, 1976).

\section{EXPERIMENT 1}

In this first experiment, a variety of Roman and braille character sets, differing along several typographical dimensions, were evaluated in terms of their tangibility and visual legibility; these eight sets are shown in Figure 1. The purpose of studying a variety of fonts for each character type was to ensure that the comparison of braille and letter tangibility did not depend on the fortuitous choice of a single representative of each. The uppercase letters of set 6 represent a compromise in height-width ratio between optimum legibility (Berger, 1944a, 1944b; McCormick, 1957) and the proportions of the standard braille cell (Nolan \& Kederis, 1969).

\section{Method}

Stimuli. The touch stimuli were produced by photoengraving. The stimuli of Figure 1 were first prepared using transfer lettering materials. The upper- and lowercase letters comprising sets 6 and 8 were taken from Letraset sheet No. 2665. Sets 4 and 5 were from Letraset sheets No. 3115 and No. 3123. The remaining sets were prepared using dots from Chartpak M 451, with the letters of set 7 corresponding in form to those of set 6 . Exact facsimiles of these 


\begin{tabular}{|c|c|c|c|c|c|c|c|}
\hline 1 & 2 & 3 & 4 & 5 & 6 & 7 & \\
\hline : & • $\because:$ & & $A N$ & $A N$ & $A N$ & ל: & \\
\hline & & : & B 0 & B 0 & $\mathrm{BO}$ & $\vdots$ & \\
\hline & ${ }^{\prime} !^{\prime}$ & & $C P$ & C P & $C P$ & : : : & \\
\hline & $\because:$ & & 00 & $D Q$ & $D Q$ & $\because \because$ & \\
\hline & $\because:$ & & E R & E R & $E R$ & $\because: \div$ & \\
\hline & $:^{\bullet}:^{\bullet}$ & $:$ & $\mathrm{FS}$ & F $\mathbf{S}$ & $F S$ & $\because \because \because$ & \\
\hline & :: : & & G T & $\mathbf{G ~ T}^{\mathrm{T}}$ & $\mathrm{G} \top$ & $\because \because$ & \\
\hline 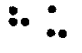 & $\therefore \therefore$ & & $\mathrm{HU}$ & H U & $\mathrm{HU}$ & $\vdots \vdots \vdots \vdots \vdots \vdots$ & \\
\hline & •" & & I V & I V & $\mathrm{IV}$ & $\vdots \because$ & \\
\hline & $\therefore \quad:$ & & JW & $J W$ & $J W$ & 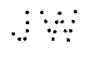 & \\
\hline 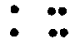 & $: \because$ & & $\mathrm{K} X$ & $K X$ & $K X$ & $\because$ & \\
\hline 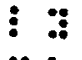 & $\vdots: \vdots$ & & $L Y$ & $L Y$ & $L Y$ & $\vdots \vdots$ & \\
\hline & $\because \because:$ & $\cdot \quad \cdots$ & $M Z$ & $M Z$ & $M Z$ & $\because \therefore$ & \\
\hline
\end{tabular}

Figure 1. The braille and Roman characters of Experiment 1 presented to the senses of touch and vision. The visual stimuli were back-illuminated transparencies subjected to optical low-pass filtering prior to viewing. The touch stimuli werc raised facsimiles presented to the distal pad of the index finger. The uppercase letters of both the transparencies and raised patterns were $5.8 \mathrm{~mm}$ in height.

characters, with the various sets arranged in concentric circles, were then embossed in zinc. The scale of the characters was such that the uppercase letters were $5.8 \mathrm{~mm}$ high. The upper surface of each character was uniformly $.8 \mathrm{~mm}$ above the etched surface of the circular plate. This metal disk, with a hole at its center, was situated horizontally so that by rotation any desired character could be positioned beneath the finger.

All stimuli were presented to the approximate center of the distal pad of the right index finger, which was held in position by a vertically mounted finger guide. Two modes of touching were used. In the static mode, the subject lightly touched the character for about $1 \mathrm{sec}$ without lateral motion. In the kinetic mode, used to prevent image fading, the finger guide was widened slightly to permit some subject-initiated motion in both longitudinal and transverse directions. The subject was instructed to make sure that the finger pad always made even contact with the entire character and the character remained roughly in the center of the pad. The purpose in so restricting movement was to prevent scanning of the edges of the character, a mode of touch that is more complex. ${ }^{2}$ Later measurements with an electronic balance indicated that subjects exerted a finger force of 5-10 g in touching the patterns.

The visual stimuli were conveniently obtained by making use of the large negative that the photoengraver used in making the contact exposure of the zinc plate. The characters were high-contrast transparent figures of precisely the same size as the touch stimuli and arranged in the same circular fashion. The negative was affixed to a Plexiglas disk and mounted vertically. Single characters were backilluminated and then blurred by diffusion; they were viewed monocularly by the subject.

On one side of the disk, a tungsten source, condensing lens, and shutter provided timed and collimated illumination of a restricted portion of the disk, with rotation and lateral displacement of the disk permitting back-illumination of any desired character. Just on the other side of the disk were mounted a pair of vertical and parallel translucent sheets of plastic. With back parallel projection of any character onto the first of these two diffusing plates, a wide range of low-pass spatial filtering could be accomplished by precisely adjusting the position of the second plate and hence the separation between them. This method of low-pass spatial filtering and its advantages are discussed by Carlson and Heyman (1979).

Pilot recognition data obtained with character sets 4 and 6 were used to determine the degree of blurring necessary to bring visual performance close to that of touch. The resulting point spread function (PSF), measured using a scanning aperture (.15 $\mathrm{mm}$ diam) in conjunction with a Spectra Spotmeter, was found to be very nearly radially symmetric. A midsection plot of the PSF is given in Figure $2 \mathrm{a}$. The corresponding two-dimensional modulation transfer function (MTF) was obtained by fitting a Gaussian to the PSF $\left(\mathrm{r}^{2}=.995\right)$ and taking its known two-dimensional Fourier transform $^{3}$; a half-midsection plot of the resulting MTF is shown in Figure $2 b$. The input patterns to this optical filter were of the same dimensions as those in the negative transparency. The filtered patterns were viewed foveally from a distance of $89 \mathrm{~cm}$ so that $1 \mathrm{~mm}$ in the image plane corresponded to a visual angle of $.064 \mathrm{deg}$. The room lights were off and scattered light was reduced to a minimum. The stimuli of $1-\mathrm{sec}$ duration were well within photopic range (the above PSF was about $1.6 \log$ units above cone threshold).

Subjects. Three sighted subjects with normal vision and ostensibly normal touch were run. J.P. and Z.L. were female, and P.C. was male; all three were in their early 20 s. None of the subjects was aware of the purpose of the experiment.
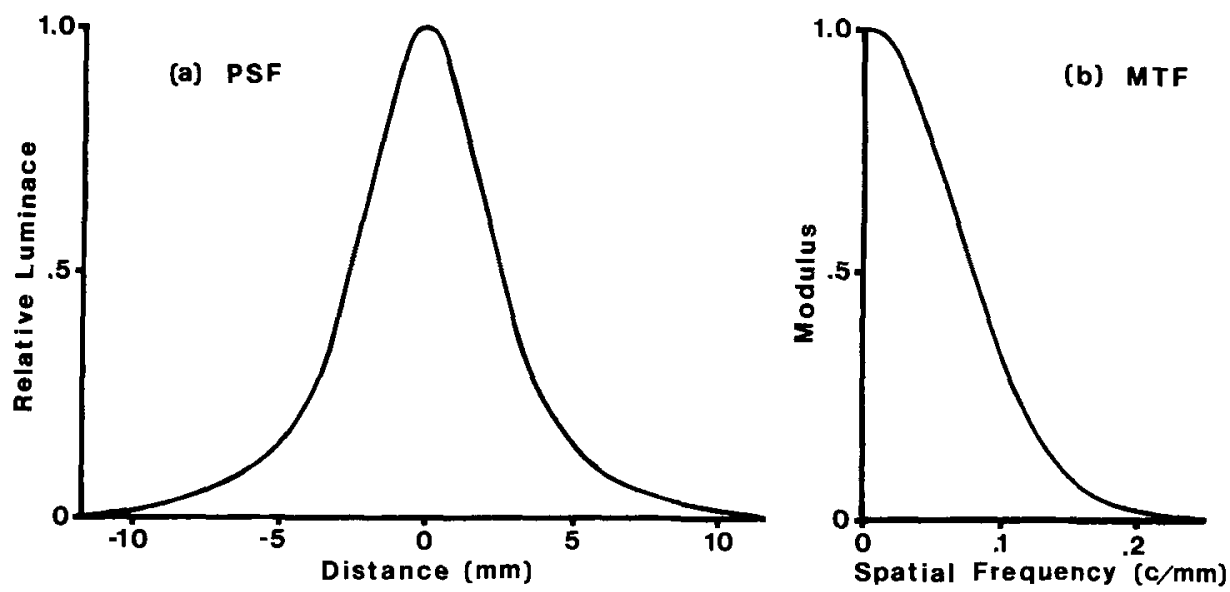

Figure 2. (a) The point spread function of the optical filter used in Experiments 1 and 3 to blur the characters of Figure 1. (b) The modulation transfer function of the optical filter; it is the Fourier transform of the two-dimensional Gaussian fit to the point spread function in (a). 
Procedure. First, each subject was taught the alphabetic characters of Standard English braille, using flashcards bearing characters based on a cell height of $7 \mathrm{~mm}$ that were viewed in focus from $65 \mathrm{~cm}$. Training continued until no identification errors were made in 10 successive runs of the alphabet. Each subject then participated in alternating sessions of vision and static touch in which the orders of character sets and characters within sets were random. Sixteen hours were required to complete four repetitions of each letter in each condition for each subject. Following this, the subject then went through 8 additional hours with kinetic touch. The subject was given feedback as to the character presented on each trial.

A second and subsidiary experiment was conducted after the main experiment. Its purpose was to obtain measures of spatial resolution for vision and touch under conditions that were the same as those in the recognition task. The acuity target employed was the König two-bar target, a configuration which enjoys certain advantages over other conventional targets such as sine-wave gratings and twopoint stimuli (Westheimer, 1977). It consists of two parallel bars, each 1 unit wide and 3 units long and separated by a 1-unit space. The tactile targets were raised patterns $(.9 \mathrm{~mm}$ embossing height) produced by photoengraving, and the visual targets were the same patterns (of equal size) from the photographic negative subjected to the same low-pass optical filter used in the main experiment. Vertical and horizontal targets of increasing size were presented to the two senses, with the subject's task being to identify its orientation; the two bars had to be resolved for orientation to be correctly judged. Threshold was defined as that bar separation, which, by interpolation, gave $75 \%$ correct performance in the twoalternative forced-choice task. The tactile task was performed only with static touch, since, with the kinetic mode of touching, cues extraneous to the spatial sense of touch (e.g., texture, resistance to finger motion) might have led to spuriously low thresholds. For both senses, 30 judgments were made at each combination of orientation and separation (from .5 to $2.0 \mathrm{~mm}$ in .5-mm increments).

\section{Results}

During the $16 \mathrm{~h}$ of testing, none of the subjects showed a substantial gain in recognition accuracy with increasing practice. Accordingly, the results given in Table 1 for character set, subject, and modality are the percentages of correct responses averaged over the four repetitions of the alphabet in each condition. Figure 3 summarizes the results for vision and static touch in the form of a scatterplot. The basic result is unequivocal-the tangibility of the different character sets is highly correlated $(r=.98)$ with their legibility under the present conditions of optical low-pass filtering. In particular, both tangibility and legibility are higher for the three braille sets than for any of the letter sets, thus lending support to the hypothesis set forth in the introduction. In the comparison of vision

Table 1

Percent Correct Recognition for Each of the Three Conditions as a Function of Subject and Character Set (CS)

\begin{tabular}{|c|c|c|c|c|c|c|c|c|c|}
\hline \multirow[b]{2}{*}{ CS } & \multicolumn{3}{|c|}{ Vision } & \multicolumn{3}{|c|}{ Static Touch } & \multicolumn{3}{|c|}{ Kinetic Touch } \\
\hline & Z.L. & J.P. & P.C. & Z.L. & J.P. & P.C. & Z.L. & J.P. & P.C. \\
\hline 1 & 76.0 & 64.4 & 57.7 & 50.0 & 48.1 & 55.8 & 60.6 & 49.0 & 79.8 \\
\hline 2 & 84.6 & 83.7 & 71.2 & 51.0 & 51.9 & 68.3 & 75.0 & 67.3 & 87.5 \\
\hline 3 & 90.4 & 90.4 & 85.6 & 70.2 & 48.1 & 75.0 & 71.2 & 56.7 & 93.3 \\
\hline 4 & 24.0 & 25.0 & 20.2 & 31.7 & 24.0 & 35.6 & 48.1 & 30.8 & 51.0 \\
\hline 5 & 27.9 & 24.0 & 21.2 & 16.3 & 23.1 & 37.5 & 45.2 & 27.9 & 60.6 \\
\hline 6 & 35.6 & 48.1 & 33.7 & 43.3 & 37.5 & 48.1 & 55.8 & 39.4 & 58.7 \\
\hline 7 & 50.0 & 50.0 & 36.5 & 34.6 & 28.8 & 51.9 & 51.9 & 33.7 & 54.8 \\
\hline 8 & 24.0 & 17.3 & 25.0 & 29.8 & 21.2 & 32.7 & 37.5 & 24.0 & 58.7 \\
\hline
\end{tabular}

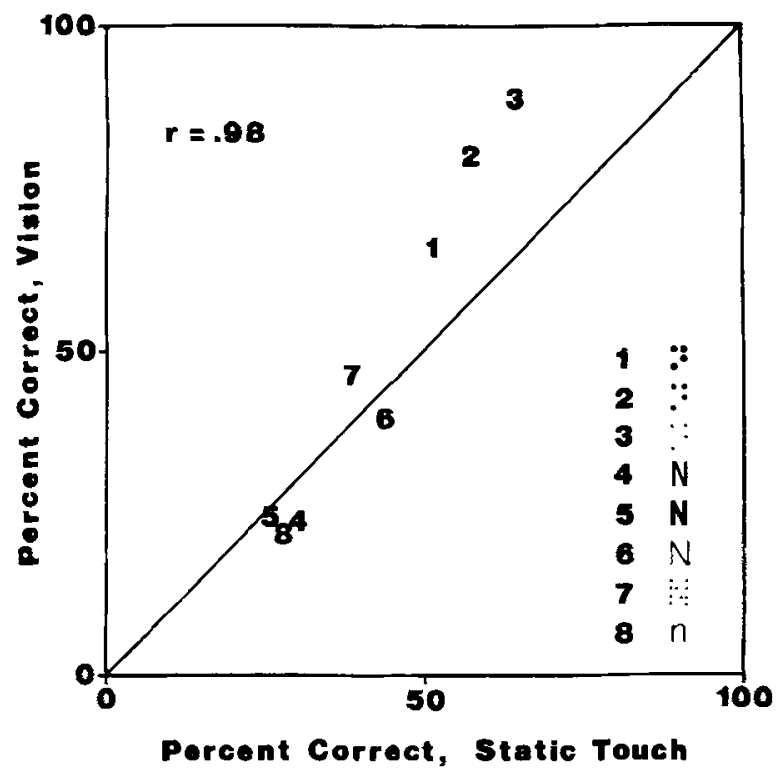

Figure 3. Scatterplot of the results of Experiment 1 for static touch and vision.

and kinetic touch, the analysis is much the same, with the value of $r$ equal to .97 .

A more detailed analysis indicated that specific characters that were highly legible for one modality were highly legible for the other. Averaging across the four uppercase letter sets and subjects, the letterspecific legibility scores (the percentages of correct recognitions) exhibited a correlation of +.87 between vision and static touch, +.83 between vision and kinetic touch, and +.89 between the two modes of touch. The corresponding correlations for the braille legibility scores (averaged over sets 1,2 , and 3 ) were $.40, .57$, and .76 , indicating somewhat poorer agreement. The correlations for condition 8 were still lower, but probably less reliable by virtue of the small sample size. Confusion errors were not analyzed because of the relatively few presentations of each character in each condition.

One might ask whether the manner in which the braille characters were sensed here is representative of normal touch reading. One indication that it is comes from considering the similarity of the braille legibility scores obtained here with those obtained by other researchers. The static touch tangibility scores exhibit a correlation of .62 with those of Bürklen (1932) (dependent variable: accuracy) and -.66 with those of Nolan and Kederis (1969) (dependent variable: threshold duration for recognition). The corresponding correlations, with kinetic touch as the comparison, were .64 and -.72 . These correlations are fair considering the value of -.57 between the measures of Bürklen and of Nolan and Kederis. However, some caution is warranted in interpreting the correlations between tangibility scores. The correlations were computed for the 26 alphabetic characters, but Bürklen (1932) and Nolan and Kederis (1969) studied other 
braille characters as well. The tangibility of a particular character depends on the characters in the set of potential stimuli with which it might be confused; strictly speaking, comparisons should be made only between sets that contain exactly the same characters. A similar point has been made by Eriksen (1980).

The spatial resolution thresholds obtained with the König bar targets were 1.7,1.7, and $1.9 \mathrm{~mm}$ for J.P., Z.L., and P.C., using static touch; the corresponding values for vision were $1.4,1.5$, and $2.0 \mathrm{~mm}$. Thus, spatial resolution proved to be well matched for the two senses under precisely the same conditions that gave rise to similar recognition performance.

\section{EXPERIMENT 2}

It might be thought that the congruence of tangibility and legibility established in the first experiment ought to be expected if visual performance is reduced to the level of tactile performance by any means whatsoever. This might be expected if it were thought that relative legibility and tangibility were functions only of the physical characteristics of the stimulus patterns and not also of the conditions of presentation and of the spatial transfer characteristics of vision and touch. This next experiment, serving as a control, showed, by counterexample, that not just any manner of reducing legibility has the equivalent effect of low-pass filtering.

\section{Method}

The characters of sets 2 and 4 (Figure 1) were mounted singly on plain index cards for tachistoscopic presentation. The biack characters exhibited a contrast of .05 relative to the uniform white surround. Viewed binocularly from a distance of $89 \mathrm{~cm}$, the characters subtended roughly $22 \times 11 \mathrm{~min}$. With long presentations, all characters were readily identifiable.

The stimulus sequence consisted of a fixation field of variable duration, the target field lasting $30,50,70$, or $90 \mathrm{msec}$, a $300-\mathrm{msec}$ masking field to halt visual processing, and a return to the fixation field between trials. The fixation field was uniform except for a just-visible fixation mark at its center. At the corresponding location, the target field contained one of the 52 letters or braille characters. The posttarget field contained at its center a highcontrast (.05) pattern mask consisting of black dots and line segments positioned randomly over an area considerably larger than that of the target characters. All fields were $11.4 \mathrm{deg}$ wide and $8.2 \mathrm{deg}$ high and were approximately $18 \mathrm{fL}$ (measured on the white surround). Field durations were calibrated by photodiode in conjunction with an oscilloscope.

The two subjects were J.P. and Z.L., both of whom had participated in Experiment 1. Prior to the recognition task, each was brought back up to criterion in the training task with the braille flashcards. In the experiment proper, they were presented each letter of the alphabet three times for each of the eight conditions (4 durations $\times 2$ character types). Trials were run in blocks of 13 with the condition fixed within each block. The orders of conditions and characters within conditions were random. Feedback was given after each trial.

\section{Results and Discussion}

Figure 4 gives the average legibility scores (percent

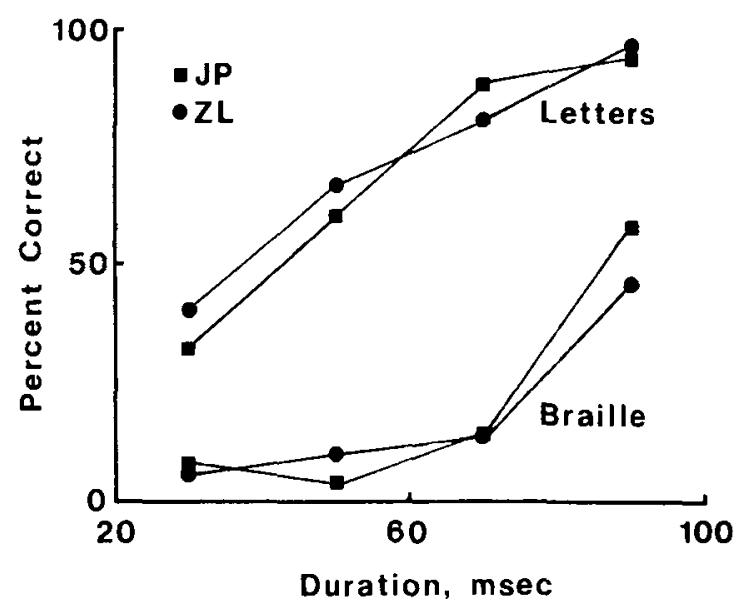

Figure 4. The results of Experiment 2.

correct) for letters and braille characters as a function of target duration. The much higher legibility of letters is obvious. This control experiment demonstrates that braille characters are not necessarily more legible than letters of comparable size with just any procedure that diminishes visual recognition performance.

Why were letters in this situation more legible than the braille characters? It is proposed here that, because of the subjects' much greater visual experience with letters, they were better able, within the limited processing times permitted by the succeeding mask, to assimilate and integrate the visual features of the letters than those of the braille characters. In Experiment 1 , however, braille characters did not suffer in this way, for processing time was more than sufficient.

The interpretation above can only be tentative, for, as Ericksen (1980) has argued recently, the use of a pattern mask is problematic. For example, in assuming that the mask interrupts visual processing, one ordinarily assumes that processing time is the same for all target characters, but this latter assumption may well be false (Eriksen, 1980). In the present work, some effort was made to devise a mask (Figure 4) that was neutral with respect to the visual interference that was thought to underlie interruption of processing. However, this goal might not have been achieved; indeed, the results obtained might have reflected a greater visual interference between the mask and the braille characters. However, for our present purpose, the reason the braille characters were selectively masked is without great consequence. Rather, this experiment demonstrates that legibility (or tangibility) is as much a function of the conditions of stimulus presentation as it is of the patterns themselves.

\section{EXPERIMENT 3}

The results of Experiment 1 lend support to the hypothesis that, after the low-pass filtering of cuta- 
neous processing, braille characters are more discriminable, and hence identifiable, than are letters. If, in fact, it is low-pass filtering which, for the most part, limits the perception of characters the size of those in Experiment 1, then increasing their size should improve their tangibility. This is so, for changing the size of a pattern amounts, in the frequency domain, to an isotropic rescaling of the two orthogonal axes of spatial frequency (Bracewell, 1978, pp. 101-103). ${ }^{4}$ Thus, increasing a character in size by a factor, $m$, is equivalent to rescaling the spatial frequency axes by the factor $1 / \mathrm{m}$, with the result that more of the spectral content of the character is passed by the fixed low-pass filter. If the optical filter in Experiment 1 were properly matched to the intrinsic filter of cutaneous processing, then the psychometric functions relating percent correct recognition to character size should be very nearly the same for tactile and visual recognition. The next experiment investigated whether or not vision and touch are affected similarly by a change in character size.

\section{Method}

Stimuli. The braille characters of set 2 (Figure 1) and the uppercase letters of set 6 were employed as the stimuli in this last experiment. After being arranged in concentric circles, they were photographed at five magnifications; the resulting negatives were then used by the photoengraver to make the zinc plates containing the raised patterns. The letters in the negatives and on the plates were about $2.8,4.3,5.7,7.2$, and $8.6 \mathrm{~mm}$ in height (relative magnifications of 1.0, 1.5, 2.0, 2.5, and 3.0). The upper surfaces of the raised characters were uniformly $.9 \mathrm{~mm}$ above the etched surface of the plate. All details of the characters were preserved, even at the smallest magnification.

In this task, a slightly different mode of touching, one that was a compromise between the static and kinetic modes of Experiment 1, was employed. Subjects made very slight circular motions of the finger as it rested on the character, taking care that there was no slippage between the corneum of the epidermis and the character. This is obviously possible to do because the subcutis, dermis, and epidermis are not rigid or rigidly abutting. The slight motion of the bulk of the finger without noticeable slippage between the stimulus and the corneum apparently causes the deforming pattern to exert a continually changing excitation of the more rapidly adapting mechanoreceptors, with the result that the tactile image does not fade. Introspectively, it would seem that this mode of touching provides no information additional to that gained from static touch at onset; it merely makes some or all of the same information available for a longer time. Using this mode of touching, subjects touched the patterns lightly for about $2 \mathrm{sec}$.

As in Experiment 1, the photographic negatives provided the stimuli for the visual task; the characters were filtered and viewed in the same way as before, except that a duration of $2 \mathrm{sec}$ was used and the optical filter adjusted such that the point spread function was $12 \%$ broader (and the MTF 12\% narrower).

Subjects. Five subjects, in their 20 s or early 30 s, participated in this experiment. Z.L. and K.H. were female, and J.L. (the author), M.M., and G.D. were male. Z.L. had participated in Experiment 1 , while the rest were new to the task and, except for J.L., were without experience as observers in touch experiments.

Procedure. As before, each subject was taught the braille alphabetic characters by means of flashcards, with the exit criterion again being 10 successive errorless runs through the cards. The subjects then participated in alternating sessions of tactile and visual recognition. M.M. and G.D. were run through four sessions of each, while K.H., J.L., and Z.L. were run through only three. In each session, every alphabetic character was tested at each of the five sizes; conditions were changed every 13 trials. Orders of conditions and of characters within conditions were random. Feedback was given after each trial.

As in Experiment 1, spatial resolution thresholds were determined following the main experiment. This time 40 , rather than 30 , trials were run at each orientation and gap size (from 1.0 to $2.5 \mathrm{~mm}$ in $.5-\mathrm{mm}$ increments). Otherwise, the procedure was precisely as before, with the mode of touching again being static touch. At the time the measurements were made, it was decided not to use the variant of kinetic touch used in the main experiment in order to prevent the intrusion of cues extraneous to the tactile sensing of form. A later control experiment with different subjects showed only a slight difference in the two modes of tbuching in the resolution task. ${ }^{3}$

\section{Results}

The results for each of the five subjects are shown in Figure 5. Each data point represents the average recognition score (percent correct) for all trials within that condition, whether based on three sessions (subjects K.H., J.L., and Z.L.) or four (M.M. and G.D.). There are several generalizations that apply to all the subjects: (1) The braille characters of set 2 are uniformly more tangible and legible than the letters of set 6; (2) increasing character size invariably results in higher recognition accuracy, regardless of condition; and (3) while visual recognition shows every sign of continuing on up to the level of errorless performance, tactile recognition tapers off at levels less than this. Incidentally, larger touch characters than those presented here were tried, but they exceeded the width of the distal pad of the finger for which good contact was possible.

Z.L.'s and J.L.'s performance on the tactile task was considerably better than that of the other three subjects. This probably reflects, in part, their considerable experience as observers in touch experiments using the distal pad of the finger. The close correspondence of the tactile and visual recognition scores, especially in the absence of a free parameter, is strong confirmation that the tactile recognition of characters in this size range is limited by the low-pass spatial filtering of cutaneous processing.

The data obtained in the spatial resolution task are given in Figure 6. The thresholds for static touch averaged $1.65 \mathrm{~mm}$ (see Footnote 5) and those for vision, $1.56 \mathrm{~mm}$. Not only are the thresholds close in value, but also the slopes of the psychometric functions are remarkably similar. However, just as important is the fact that these similar psychometric functions were obtained under very nearly the same conditions that led to a congruence of the visual and tactile recognition scores for two of the subjects.

\section{GENERAL DISCUSSION}

Among other things, Experiments 1 and 3 confirm that the braille alphabetic characters, as a set, are more tangible than upper- and lower-case Roman letters. 

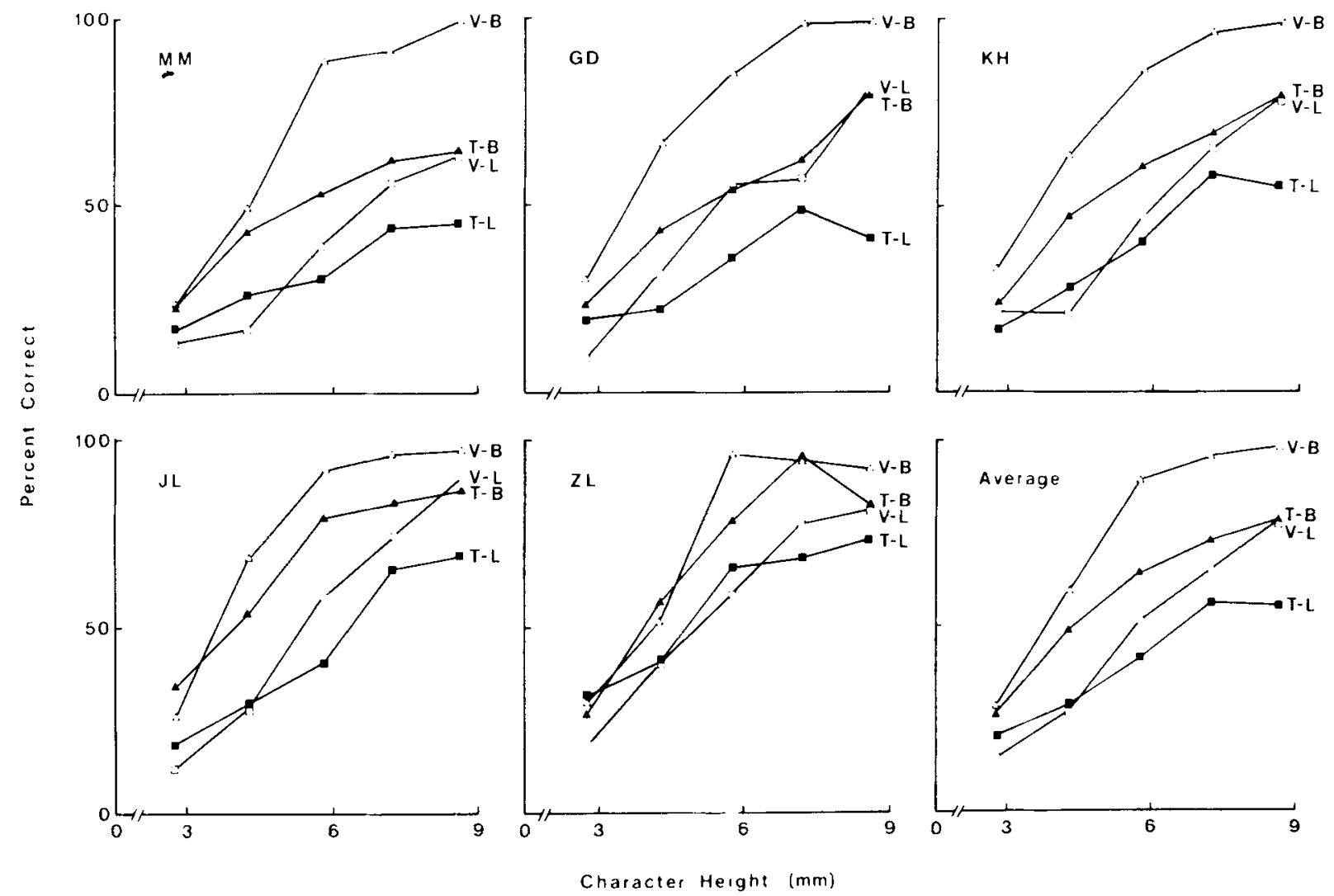

Figure 5. The results of Experiment 3. $V=$ vision, $T=$ touch, $L=$ letters, and $B=$ braille.

How can this fact be explained? The hypothesis that motivated the present work was that, after the lowpass filtering of cutaneous processing, braille characters comprise a more discriminable set of characters than do Roman letters. There are, however, other interpretations that merit consideration as well.

Merry (1937) suggested that raised dots were more palpable than smooth raised lines. A natural extension of this idea would be that characters composed of dots might be more tangible than characters composed of line segments. Some of the early experimentation with raised print included attempts to enhance the tangibility of the letters by forming them with dots (Bledsoe, 1972; Bürklen, 1932; Merry, 1937), reportedly with some success (Bürklen, 1932, p. 3). It is certainly worthwhile to consider that braille might owe its superior tangibility to the punctateness of its stimulation.

Another interpretation might be that braille characters are simply more variable than letters in terms of global or overall shape (Bürklen, 1932; Nolan \& Kederis, 1969). Evaluating this idea is problematic, for "overall shape" is not a well-defined notion. On the one hand, overall shape might be just another way of referring to the lower spatial frequency content of a pattern, in which case this interpretation simply would reduce to the earlier hypothesis. On the other hand, overall shape might refer to something quite different, such as the smoothed perimeter of a spatial pattern (with zero weight given to the internal structure). In its normal usage, the term probably has a multiplicity of meanings. Though vague, the notion of overall shape remains appealing. When one compares the filtered versions of the same pattern after low-pass filtering and after high-pass filtering, the two versions, even when they have nonoverlapping spectra, share an aspect in common - the characteristic, or overall, shape of the pattern. This very fact is reason enough for not identifying the overall shape of a pattern simply with its lower spatial frequency content. However, wide-band patterns differing in this ill-defined sense of overall shape will also generally differ, after low-pass filtering, in terms of the remaining spectral content; thus, the filtering and overall shape interpretations cannot be considered mutually exclusive.

The basic thrust of Experiments 1 and 3 is that when the visual stimuli (characters and resolution targets) are optically low-pass filtered to such a degree that the indices of tactile and spatial resolution are closely matching, visual recognition mimics tactile recognition. Since the optical stimuli are known to be low-passfiltered characters (by reason of the experimental oper- 


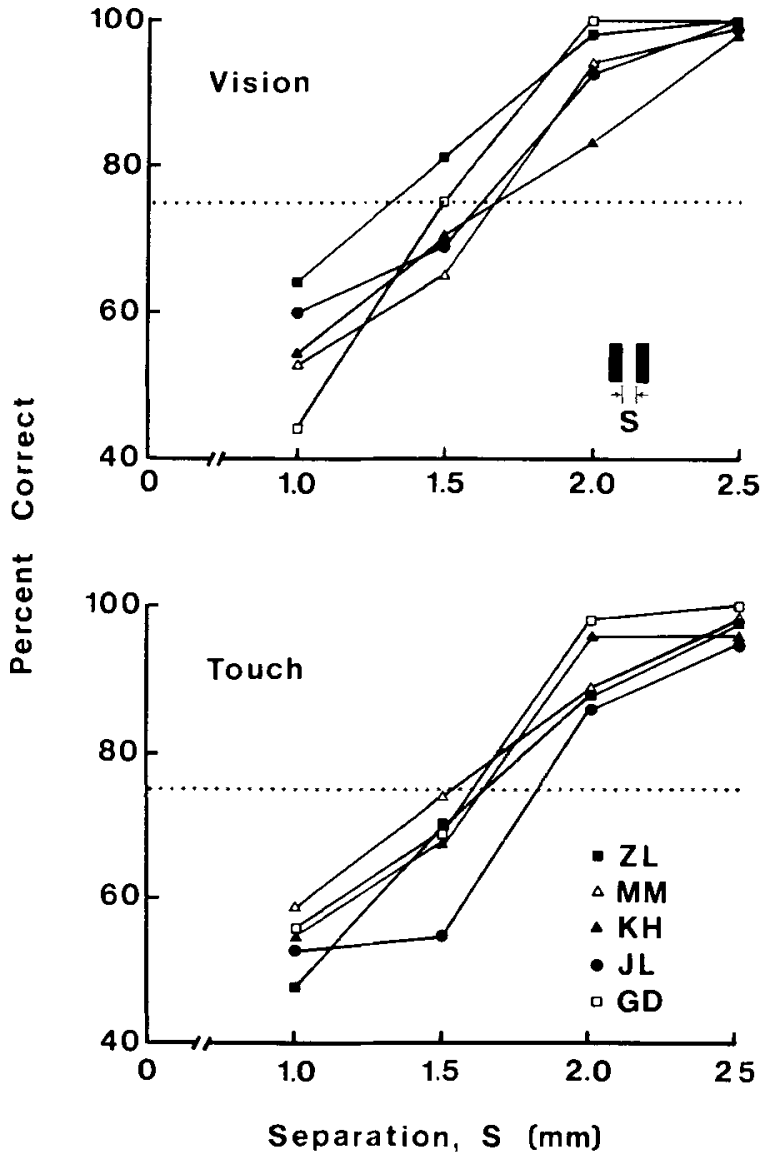

Figure 6. Results of the spatial resolution task for the subjects of Experiment 3. Percentage of correct judgments of the König target orientation is plotted as a function of the bar separation.

ation), and since the presumption is strong that the touch stimuli are low-pass filtered in the course of cutaneous processing (they must be if the spatial resolution task is to mean anything), the congruence of the tactile and visual recognition data strongly suggests that tactile recognition of characters under these conditions is largely determined by their low-frequency content. In particular, braille characters must be more variable in their low-frequency content than letters, since they are uniformly more recognizable for both senses when low-pass filtering is effectively limiting performance (see Figure 7).

In addition, Experiment 1 poses a problem for the interpretation based on the notion of overall shape. There are probably few definitions of overall shape on the basis of which one would maintain that the same braille characters from each of sets 1,2 , and 3 differ much in overall shape. Yet, these sets vary considerably in terms of both tangibility and legibility, with lowpass filtering the obvious controlling factor. In general, for legibility and tangibility to be fully understood, consideration must be given not only to the patterns themselves, but also to the conditions under which they are presented (cf. Tinker, 1963) and to the spatial and temporal transfer properties of the senses (cf. Bouma, 1971); this point is nicely illustrated by the widely differing results on the legibility of letters and braille found in Experiments 1 and 2.

Although the spatial-filtering hypothesis receives strong support in this work, there may be circumstances in which the notion of overall shape, despite its vagueness, might prove useful as an explanatory concept. Consider, for example, the possibility that braille characters based on a cell height of $20 \mathrm{~mm}$ could be more tangible than letters of the same height when scanned by the fingertip. One would not expect the neural representations of characters this large to suffer much loss in fidelity as a result of the filtering of cutaneous processing. ${ }^{6}$ If such a result were obtained, it might be necessary to invoke the concept of overall shape. The argument would continue that braille characters, regardless of size, are more dissimilar in overall shape than are letters and that, when reduced to a size such that the higher spatial frequencies are filtered out in the cutaneous transfer, they still remain more dissimilar in terms of the spectral content that remains. This argument is certainly plausible, but it suffers from the vagueness of the notion of overall shape. In contrast, the spatial filtering idea is well defined, though limited in its domain of application. It is also strongly supported by the results of the present work; moreover, in view of the fact that embossed braille characters are approximately the height and width of those in Experiment 1 and squarely in the middle of the size range used in Experiment 3, the filtering explanation surely includes ordinary braille within its domain.

Given the confirmation of the spatial filtering hypothesis, what contribution might the punctate nature of braille make to its tangibility? To discuss

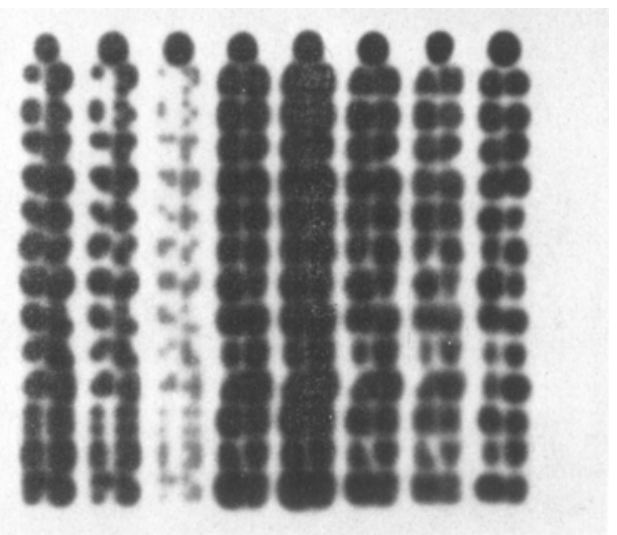

Figure 7. A low-pass filtered version of Figure 1 created by placing a diffusing plate in the enlarger between the photographic paper and the negative of Figure 1. Note the correspondence between the relative discriminability of the different character sets and the results for static touch (Figure 3). 
this, we need to distinguish between the fact that braille characters are formed from a 2 by 3 array of dots and the possibility that the punctate quality of the deforming stimulus might enhance its excitation of the mechanoreceptors and subsequent form-analyzing mechanisms. It is the former which accounts for the greater shape variation of braille characters (re letters) and thus their greater variation in the spectral content that remains after low-pass filtering. It is the latter to which our immediate concern is directed. The comparisons of sets 6 and 7 for both static and kinetic touch indicated that punctographic letters were no more tangible than conventional letters, thus replicating the finding by Austin and Sleight (1952); this was so even though the punctateness was quite perceptible to the subjects, especially with kinetic touch. A second fact is that in both Experiments 1 and 3 the edge that braille characters enjoyed over letters in legibility was greater than the edge in tangibility; if the punctate quality of braille were to facilitate tactile recognition, the reverse would have been expected. Tentatively, one might conclude that making the elements of a raised pattern more palpable by serrating them or by otherwise enhancing their texture does not facilitate recognition of the form.

Although the results of Experiments 1 and 3 lend strong support to the filtering hypothesis, they also point to another factor that limits the tactile sensing of form. Even for the two most proficient subjects in Experiment 3, the improvement in tactile recognition with increasing character size (both letters and braille) shows signs of asymptoting at less than perfect levels, while that in visual recognition does not. For Subjects M.M. and G.D., who are much less proficient in the tactile task, the psychometric functions asymptote at still lower levels. Part of the reason that tactile recognition falls short of visual recognition might be that the largest characters fail to make uniform contact with the rounded finger pad; this appears to be true for Subject Z.L., who scored consistently higher in the sessions with braille characters that were $7.2 \mathrm{~mm}$ high than in those with braille characters that were $8.6 \mathrm{~mm}$ high. Yet, more is involved, for the disparity between touch and vision for the larger characters varies considerably with subject. It is reasonable to suppose that there is sufficient fidelity of the sensory information arriving at the somatosensory cortex with the large letters but that this information is not being properly integrated by the subject. Kirman (1973 and Note 1) has discussed at some length the limiting role of perceptual integration in tactile pattern perception. That the stage of perceptual integration might be limiting is supported by the author's impressions as a subject; in the tactile task there were numerous occasions on which the difficult features of a stimulus character could be sensed, but their spatial relationship seemed equivocal. Further support for this idea derives from the fact that the two most proficient subjects were those who had had prior experience as observers in experiments dealing with tactile pattern perception. It is likely that, as subjects learn to integrate the sensory information with practice, their performance is limited more and more exclusively by the spatial sensitivity of touch. In all of this work, however, the patterns have been simple ones. Surely one would not expect that the sense of touch, which lacks the specialized neural machinery that vision has for the processing of form, could ever equal vision in the perception of intricate spatial patterns, even under conditions that avoided the loss of detail through cutaneous spatial filtering.

A look back at two of the major studies on braille lends some additional support to the present interpretation of the superior tangibility of braille. Bürklen (1932) investigated the accuracy with which the various braille characters could be identified. Unfortunately, he worked with characters approximately twice the height and width of standard braille; having obtained few errors in recognition with such large characters, he found it necessary to reduce their tangibility by covering the fingertips of the subjects with rubber caps. Given these unrepresentative conditions, his findings must be treated with skepticism. Bürklen used his analysis of the relative tangibility of the different characters and of the confusions between them to support his view that "characteristic shape" was most important in braille recognition, a view that, from his discussion, appears to have been molded in part by the writings of earlier researchers and the introspections of braille readers. Although Bürklen did not fully articulate what he meant by "characteristic shape," it is apparent from the following quotations that he anticipated the present reasoning in terms of spatial filtering:

After braille reading has been learned, the dots are no longer considered, but attention is concerned with recognizing the symbols as a whole, as characteristic touch images (Bürklen, 1932, p. 19).

The fact that two points which are only a short distance apart are difficult to separate from one another and that they seem to be bound together as by a bridge, seems to prove the assumption that in touch reading the dots of one character cannot be felt separately but that a unit picture of the characters is perceived and that, therefore, the shape of the characters is of decided importance for comprehension. A fusion of dots by lines, as proposed by Kunz and Javal for increasing the tangibility, thus seems superfluous (Bürklen, 1932, pp. 49, 50).

Having greater face validity than the work of Bürklen is the more recent investigation of Nolan and Kederis (1969). They presented braille characters of normal size for short durations using a device called a tachis- 
totactometer and defined tangibility in terms of the threshold duration for recognition. Although there were several significant differences between their findings and Bürklen's, they concurred on the importance of shape as a determinant of tangibility; they found that roughly three-fifths of the confusion errors common to at least $25 \%$ of their subjects were on characters similar in shape to the characters presented. They found also that tangibility was inversely related to the number of dots defining the character and positively related to the size of the spaces between the dots that were present. These findings are consistent with the spatial filtering hypothesis.

Finally, because it has been shown here that braille characters under low-pass filtering are more legible than letters, it could be thought that printing books and other material in braille rather than ordinary type might facilitate visual reading. This expectation has no basis in fact, for Wallsten and Lambert (Note 2) have found that highly proficient sighted readers of braille read visual braille at much lower rates than they do normal type, even when the braille and type are equated in terms of contrast and angular size. At normal reading distances, the braille and Roman characters would not have suffered any loss in their critical detail as a consequence of the filtering properties of foveal or parafoveal vision. Thus, one might argue that line-stroke characters are more suited to processing by the eye than are punctographic characters. Braille might conceivably prove advantageous as a medium for visual communication under those circumstances in which characters suffer a significant loss in fidelity as a result of low-pass filtering by the eye, such as when road signs are viewed from a great distance. The relevance of Wallsten and Lambert's finding to the present work is in showing, as did Experiment 2, that legibility is situation-specific (also cf. Tinker, 1963), depending as much on task variables and transfer characteristics of vision as on the stimuli themselves.

\section{REFERENCE NOTES}

1. Kirman, J. H. Tactile pattern perception and tactile displays. Paper presented at the Conference on Interrelations of the Communicative Senses, Asilomar, California, September 29-October 2, 1978.

2. Wallsten, T. S., \& Lambert, R. M. Visual braille and print reading as a function of display field size. Manuscript in preparation.

\section{REFERENCES}

Apkarian-Stielau, P., \& Loomis, J. M. A comparison of tactile and blurred visual form perception. Perception \& Psychophysics, 1975, 18, 362-368.

Austin, T. R., \& Sleight, R. B. Accuracy of tactual discrimination of letters, numerals, and geometric forms. Journal of Experimental Psychology, 1952, 43, 239-247.
BERGER, C. I. Stroke-width, form, and horizontal spacing of numerals as determinants of the threshold of recognition. Journal of Applied Psychology, 1944, 28, 208-231. (a)

BERGER, C. II. Stroke-width, form, and horizontal spacing of numerals as determinants of the threshold of recognition. Journal of Applied Psychology, 1944, 28, 336-346. (b)

Bledsoe, W. Braille: A success story. In Evaluation of sensory aids for the visually handicapped. Washington, D.C: National Academy of Sciences, 1972.

BoumA, H. Visual recognition of isolated lower-case letters. Vision Research, 1971, 11, 459-474.

BraCEWELL, R. N. The Fourier transform and its applications. New York: McGraw-Hill, 1978.

Breitmeyer, B. G., \& Ganz, L. Implications of sustained and transient channels for theories of visual pattern masking, saccadic suppression, and information processing. Psychological Review, 1976, 83, 1-36.

BürKLEN, K. Touch reading of the blind (F. K. Merry, trans.). New York: American Foundation for the Blind, 1932.

Camprell, F. W.. \& Robson, J. G. Application of Fourier analysis to the visibility of gratings. Journal of Physiology, 1968, 197, 551-556.

Carlson, C. R., \& Heyman, P. M. A large format optical display for the generation of generalized psychophysical stimuli. Vision Research, 1979, 19, 99-103.

Craig, J. C. A confusion matrix for tactually presented letters. Perception \& Psychophysics, 1979, 26, 409-411.

Eriksen, C. W. The use of a visual mask may seriously confound your experiment. Perception \& Psychophysics, 1980, 28, 89-92.

Gilmore, G. C., Hersh, H., Caramazza, A., \& Griffin, J. Multidimensional letter similarity derived from recognition errors. Perception \& Psychophysics, 1979, 25, 425-431.

Goodman, J. W. Introduction to Fourier optics. New York: McGraw-Hill, 1968.

KIRMan, J. H. Tactile communication of speech: A review and an analysis. Psychological Bulletin, 1973, 80, 54-74.

Loomis, J. M., \& Apkanian-Stielau, P. A lateral masking effect in tactile and blurred visual letter recognition. Perception \& Psychophysics, 1976, 20, 221-226.

Lowenfeld, B., Abel, G. L., \& Hatlen, P. H. Blind children learn to read. Springfield, Ill: Thomas, 1969.

McCormick, E. J. Human engineering. New York: McGrawHill, 1957.

MerRy, R. V. Fingers for eyes. The story of raised print. Scientific Monthly, 1937, 44, 273-279.

Meyers, E., Ethington, D., \& Ashcroft, S. Readability of braille as a function of three spacing variables. Journal of Applied Psychology, 1958, 42, 163-165.

Nolan, C. Y., \& Kederis, C. J. Perceptual factors in braille word recognition. New York: American Foundation for the Blind, 1969.

Tinker, M. A. Legibility of print. Ames: Iowa University Press, 1963.

WEINSTEIN, $S$. Intensive and extensive aspects of tactile sensitivity as a function of body part, sex, and laterality. In D. R. Kenshalo (Ed.), The skin senses. Springfield, 11l: Thomas, 1968.

WESTHE IMER, G. Spatial-frequency and light-spread descriptions of visual acuity and hyperacuity. Journal of the Optical Society of America, 1977, 67, 207-212.

\section{NOTES}

1. Because the cutaneous transfer of spatial patterns from stimulus to cortical representation discards the higher spatial frequencies, as do all real-image processing systems, it is appropriate to refer to this aspect of cutaneous processing as low-pass spatial filtering. Similarly, the information about each pattern that remains after filtering is aptly referred to as the low spatial frequency content. 
By no means, however, is it intended that the information about each pattern is represented in the spatial frequency domain, either by narrow-band or by broad-band spatial frequency channels. The spatial filtering hypothesis considered in this paper is neutral with regard to how spatial patterns are represented neurally.

2. In normal tactile exploration of a finely detailed pattern, a person deliberately makes contact with only a portion of the pattern at a time, often by using the convex surface of the fingertip or by tilting the finger. In braille reading, the reader moves the finger across the character so that first the left edge, then the entire character, and finally the right edge are in contact with the finger. For an image processing system greatly limited in spatial bandwidth, scanning can be advantageous, since the amount of image spread, and hence the degree of lateral masking, can be reduced (Loomis \& Apkarian-Stielau, 1976). To properly simulate tactile sequential scanning with blurred vision, one would need to work with a visual display that permitted the subject to scan the character with an aperture.

3. If $x$ and $y$ are orthogonal spatial dimensions and $\mathrm{c}$ is a scaling factor, then the two-dimensional Gaussian exp $-\pi\left[\left(\mathrm{x}^{2} / \mathrm{c}^{2}\right)+\right.$ $\left.\left.\left(y^{2} / c^{2}\right)\right]\right\}$ has the two-dimensional Fourier transform $c^{2} \exp [-\pi$ $\left.\left(c^{2} u^{2}+c^{2} y^{2}\right)\right]$, where $u$ and $v$ are orthogonal dimensions of spatial frequency (Bracewell, 1978). The modulation transfer function is the modulus (amplitude function) of the normalized transfer function (Goodman, 1968), which in this case is real and even and thus equals its modulus.

4. In addition to the rescaling of the frequency axes of the Fourier transform of a pattern when it is changed in size, there is a rescaling of its amplitude function: By the similarity theorem (Bracewell, 1978, pp. 101, 244), if $\mathrm{f}(\mathrm{x}, \mathrm{y})$ has the Fourier transform $F(u, v)$, then $f(a x, a y)$ has the transform $\left(a^{2}\right)^{-1} F(u / a, v / a)$.

5. Resolution thresholds of $1.32,1.44$, and $1.67 \mathrm{~mm}$ were obtained for three subjects using the mode of touch of Experiment 3; the corresponding thresholds for static touch were $1.53,1.63$, and $1.74 \mathrm{~mm}$. The average drop in threshold of $.15 \mathrm{~mm}$ is insignificant when compared with the enormous variation in spatial resolution (measured with the two-point target) across body loci (Weinstein, 1968). Had body locus also been a variable in the present experiment, the recognition psychometric functions, with character size the independent variable, would presumably have shifted along the size axis a distance comparable to the change in spatial resolution.
6. Attention must be paid to the conditions that determine the variation in tangibility or legibility across a set of characters. Craig (1979) has shown that letters much larger than those studied here, when briefly presented to the finger with the vibrotactile display of the Optacon, exhibit a variation in tangibility quite like the variation in legibility of briefly presented letters (Gilmore, Hersh, Caramazza, \& Griffin, 1979). Prima facie, this would suggest that something other than lower spatial frequency content accounts for the common variation in tangibility and legibility, since in neither experiment were the characters ostensibly subjected to any low-pass filtering. Although this conclusion may be correct, it cannot be assumed, for it is conceivable that the experimental conditions in both studies did result in effective low-pass filtering of the letters. With regard to the visual task, consider the effect of reducing the exposure duration of letters that at long durations are readily perceived. Without a succeeding pattern mask, reducing exposure duration below the critical duration of Bloch's law amounts to a lowering of stimulus contrast. Because the contrast sensitivity function of foveal vision at photopic levels (Campbell \& Robson, 1968) manifests the band-pass filtering of visual processing, lowering stimulus contrast (directly or indirectly) causes a reduction in bandwidth of the processed image; this is so because only the spatial frequency content of a pattern near the peak of the contrast sensitivity function is passed by the visual system when stimulus contrast is low. If, in addition, reducing exposure duration has the effect of shifting spatial sensitivity to lower frequencies as might occur with a partial transition from sustained to transient cells (Breitmeyer \& Ganz, 1976), the effective low-pass filtering by the visual system would be all the more pronounced. A similar argument might be advanced for touch as well. Observe that the effect of reducing target duration without a succeeding pattern mask is likely to be quite different from that when a pattern mask does follow (see Discussion of Experiment 2). The point of this discussion is to show that considerable reflection is warranted before one can presume which stimulus properties and which transfer characteristics of the sense in question determine the observed pattern of tangibility or legibility.

(Received for publication June 12, 1980; accepted revision received October 26, 1980.) 